\title{
Résumé : Évaluation, à l'aide de l'imagerie satellite, des risques de santé publique posés par la contamination microbienne des eaux récréatives
}

\author{
Turgeon $\mathrm{P}^{1 *}$, Brazeau $\mathrm{S}^{1}$, Kotchi SO ${ }^{1}$, Pelcat $\mathrm{Y}^{1}$ et Michel $\mathrm{P}^{1}$ \\ ${ }^{1}$ Laboratoire de lutte contre les zoonoses d'origine alimentaire, Agence de la santé publique du Canada, \\ Saint-Hyacinthe (Québec) \\ *Auteure-ressource : patricia.turgeon@phac-aspc.gc.ca
}

\section{Résumé}

Contexte : La contamination fécale des eaux récréatives peut provoquer des gastroentérites, des infections respiratoires, des dermatites et des infections de l'oreille. Outre la réalisation de tests directs de contamination des eaux, l'Organisation mondiale de la Santé (OMS) recommande, dans le cadre des activités de surveillance, l'évaluation des facteurs environnementaux connus pour agir sur la qualité de l'eau. La mesure de ces facteurs à l'aide de l'imagerie satellite peut se révéler utile au Canada dans les cas où il faut surveiller de grandes superficies ou des endroits difficiles d'accès.

Objectif : Évaluer la valeur ajoutée que confère l'utilisation de l'imagerie satellite pour surveiller et gérer les risques microbiens associés aux eaux récréatives au Canada.

Méthodes : On a utilisé des images satellites pour calculer cinq indices environnementaux qui peuvent avoir une incidence sur le risque de contamination des eaux récréatives : les terres agricoles, les zones urbaines (surfaces imperméables), la forêt et les zones humides. On a ensuite comparé les modèles statistiques incluant ces indices avec le niveau de contamination moyen des plages du sud du Québec, au Canada. On a évalué divers capteurs satellitaires à l'aide de critères d'exactitude et de performance.

Résultats : La classification des images satellites a donné de bons résultats dans la zone d'étude. Pour deux de ces variables, à savoir les terres agricoles et les zones urbaines, la concentration en coliformes était sensiblement plus élevée. Dans le cadre de cette évaluation, c'est le capteur Landsat-5 qui offrait le meilleur rapport coûts-avantages.

Conclusion : L'imagerie satellite peut servir à déterminer les facteurs environnementaux associés à un risque accru de contamination fécale des eaux récréatives au Canada et peut venir compléter les activités actuelles de surveillance et d'évaluation des risques.

\section{Introduction}

La baignade et les activités en eaux naturelles peuvent présenter divers risques pour la santé humaine, notamment celui de maladies infectieuses. La gastroentérite est la maladie infectieuse la plus communément associée à la baignade, même si on a aussi établi un lien entre les infections respiratoires, les dermatites et les infections de l'oreille d'une part, et une mauvaise qualité de l'eau d'autre part (1-4).

À l'heure actuelle, la principale stratégie utilisée pour surveiller la qualité de l'eau est la détection d'indicateurs microbiens de pollution fécale (tels que les coliformes fécaux dans les échantillons d'eau). Ces mesures sont considérées comme nécessaires, mais l'OMS recommande également de surveiller les sources et les 
caractéristiques environnementales qui peuvent avoir une incidence sur la qualité de l'eau pour évaluer ces risques de façon globale. Combinées, ces deux méthodes pourraient fournir des données de base permettant de classer les plages en fonction du risque de pollution et du niveau réel de contamination fécale (1).

La télé-épidémiologie emploie des technologies spatiales telles que l'imagerie satellite en combinaison avec une stratégie épidémiologique pour surveiller et évaluer la répartition des maladies animales et humaines liées aux variations climatiques et environnementales (5). L'imagerie satellite peut fournir de l'information fiable sur les caractéristiques du sol pour de grandes superficies et à ce titre, constitue un formidable atout pour l'évaluation globale de la contamination fécale des eaux récréatives (6). Étant donné que le Canada compte des milliers de lacs facilement accessibles à une grande proportion de la population et qu'ils sont plus souvent utilisés à des fins récréatives, nous voulions évaluer la valeur ajoutée qu'apporte la télé-épidémiologie pour surveiller efficacement les risques microbiens que représentent ces eaux dans un contexte canadien.

Les indices obtenus grâce aux données satellites ont été inclus dans les modèles statistiques, puis ils ont été comparés au niveau moyen de contamination de plusieurs plages du sud du Québec. En plus d'évaluer l'efficacité de cette méthode, nous avons également comparé divers types d'imagerie satellite du point de vue opérationnel. Les détails techniques de cette étude se trouvent dans un rapport plus complet (7).

\section{Méthode}

\section{Zone d'étude}

La zone d'étude comprenait trois bassins hydrographiques du sud du Québec, à savoir ceux des rivières

Yamaska, Saint-François et l'Assomption. On a inclus dans les analyses les plages publiques adjacentes à un lac et surveillées par le programme de surveillance des eaux récréatives du Québec pendant au moins trois étés entre 2004 et $2011(n=78)$ (Figure 1)(8). 
Figure 1 : Répartition des plages étudiées dans les trois bassins hydrographiques du sud du Québec.

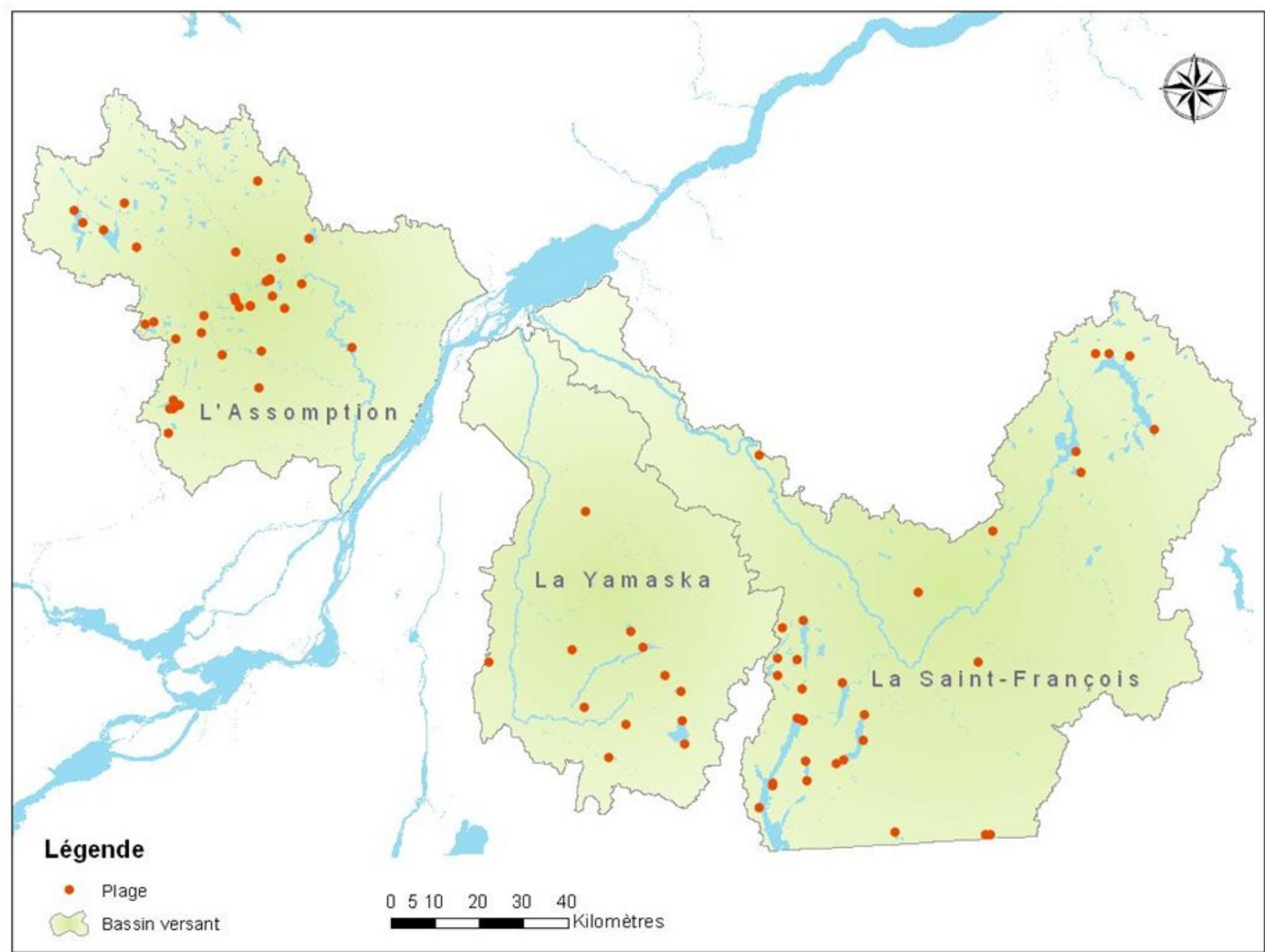

\section{Mesure de la contamination fécale}

Les mesures de la contamination fécale proviennent d'Environnement-Plage, le programme de surveillance des eaux récréatives du Québec. Conformément à ce programme, des échantillons d'eau ont été prélevés en juin, en juillet et en août chaque été pendant la période d'étude (entre 2004 et 2011). Une description détaillée de ce programme est disponible (9). Aux fins de la présente étude, on a utilisé la moyenne géométrique des concentrations de coliformes fécaux mesurées dans l'ensemble des échantillons prélevés au cours de la période de l'étude comme indice représentant le niveau moyen de contamination de chaque plage. Cette mesure se voulait être un indicateur général de la contamination d'une plage donnée qui serait très peu influencé par les valeurs extrêmes pouvant découler de circonstances particulières comme de fortes pluies ou des vagues de chaleur.

\section{Facteurs environnementaux}

Il existe deux grands types de facteurs dont l'incidence sur la contamination fécale des eaux récréatives naturelles (et donc sur leur qualité) est connue : les conditions météorologiques ( $p$. ex. les précipitations et la température), qui peuvent varier selon le moment où l'échantillonnage est réalisé, et les caractéristiques environnementales (p. ex. la topographie et l'utilisation des terres), qui sont relativement stables à long terme 
pour une plage (10-14). Dans le cadre de ce projet, on a examiné les facteurs environnementaux associés à la contamination fécale des eaux récréatives qui restent stables au fil du temps (15).

On a extrait quatre facteurs liés à l'utilisation des terres et à la couverture terrestre des images satellites (terres agricoles, surfaces imperméables, forêts et zones humides). Les terres agricoles comprennent les zones cultivées et les pâturages. On sait que ces terres ont un effet sur la qualité de l'eau en aval, car elles peuvent être une source de micro-organismes fécaux provenant de l'épandage de fumier ou des animaux au pâturage $(16,17)$. Les surfaces imperméables ont été utilisées comme substitut des zones urbaines. Ces zones peuvent être liées à la pollution de l'eau par les matières fécales par les rejets du système de traitement des eaux usées et les écoulements urbains (13). À l'inverse, les forêts et les zones humides sont associées à une meilleure qualité de l'eau, car elles filtrent les micro-organismes (18-20). On a également ajouté à l'analyse quatre autres facteurs associés à des caractéristiques géohydrologiques précises des plages qui n'ont pas été extraits des images satellites : la topographie, le nombre d'affluents, l'environnement des lacs et un indice de rusticité des plantes produit par Environnement Canada, ce dernier servant à représenter les conditions climatiques moyennes de la région $(21,22)$.

\section{Estimation des facteurs environnementaux grâce à la classification des images satellites}

On a utilisé différents capteurs satellitaires pour extraire des données sur l'utilisation des terres : SPOT-5, Landsat 5 , Meris FRS 1, un spectroradiomètre imageur à résolution moyenne (MODIS), un radiomètre perfectionné à très haute résolution (AVHRR), GeoEye-1 et WorldView-2. La Figure 2 illustre les étapes suivies pour transformer les images satellites brutes en images classées pouvant être utilisées aux fins d'analyses. Chaque capteur offre des fonctionnalités précises qui peuvent avoir un effet sur leur capacité à évaluer les caractéristiques environnementales associées à un niveau accru de contamination fécale des eaux récréatives. Dans l'hypothèse d'une utilisation dans le cadre d'un programme de surveillance, nous avons également examiné les critères opérationnels, dont le coût des images, les heures de travail, le niveau d'expertise et le matériel requis pour traiter les images, ainsi que des critères liés à l'adéquation et à l'efficacité des analyses statistiques représentant l'association entre les niveaux de contamination et les facteurs environnementaux. 
Figure 2 : Schéma de la méthodologie générale de traitement des diverses images satellites utilisées pour l'évaluation.

Une fois les images obtenues, ce processus consiste notamment à corriger et à étalonner les images, ainsi qu'à sélectionner les sites qui permettront de réaliser la classification des images. On utilise les images classées représentant diverses couvertures terrestres pour extraire les données, puis pour réaliser les analyses épidémiologiques.

\section{Correction et étalonnage de l'image satellite}

\section{a. Image satellite non classée}
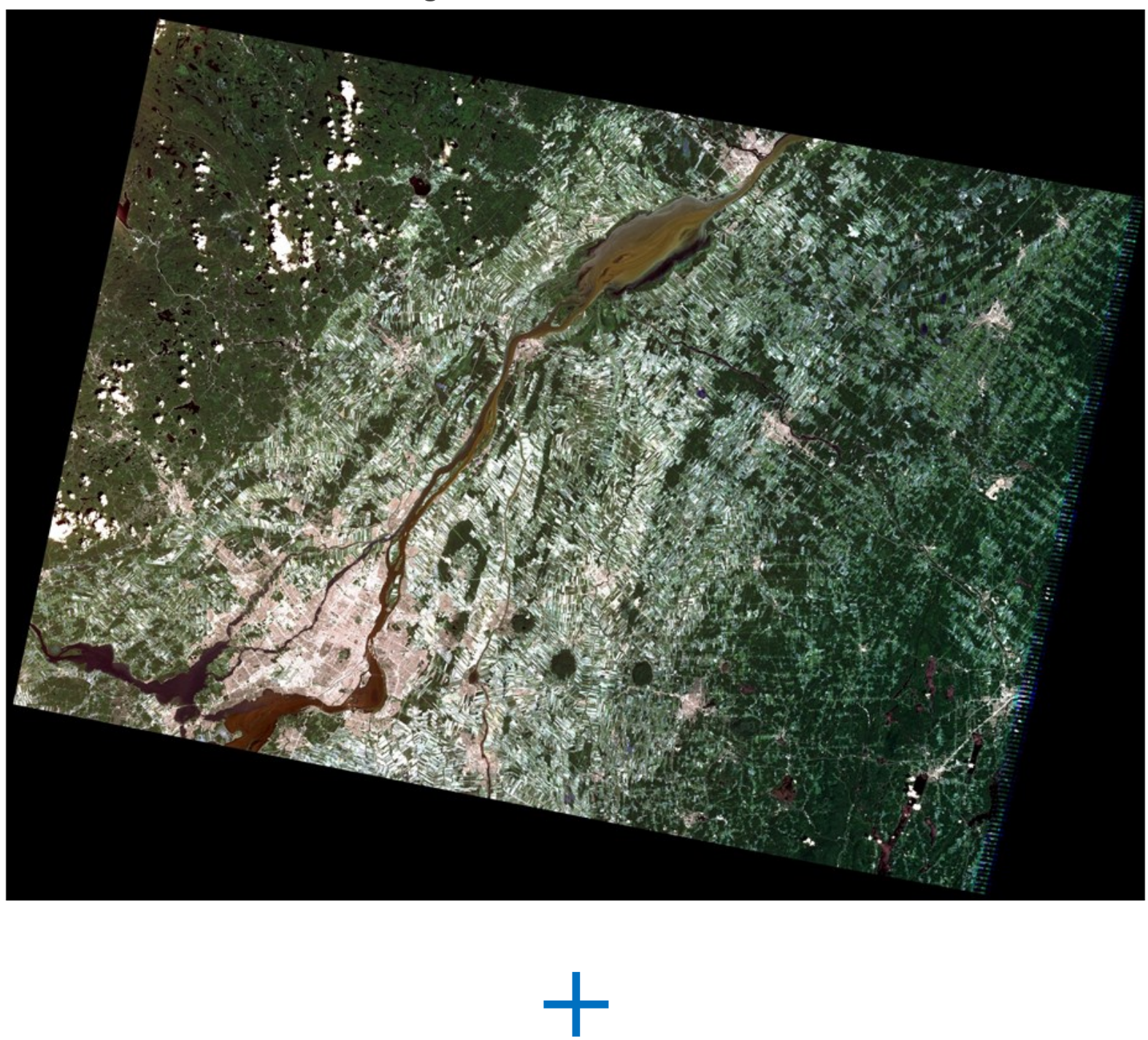


\section{b. Sites sélectionnés pour déterminer et caractériser les surfaces à classer}
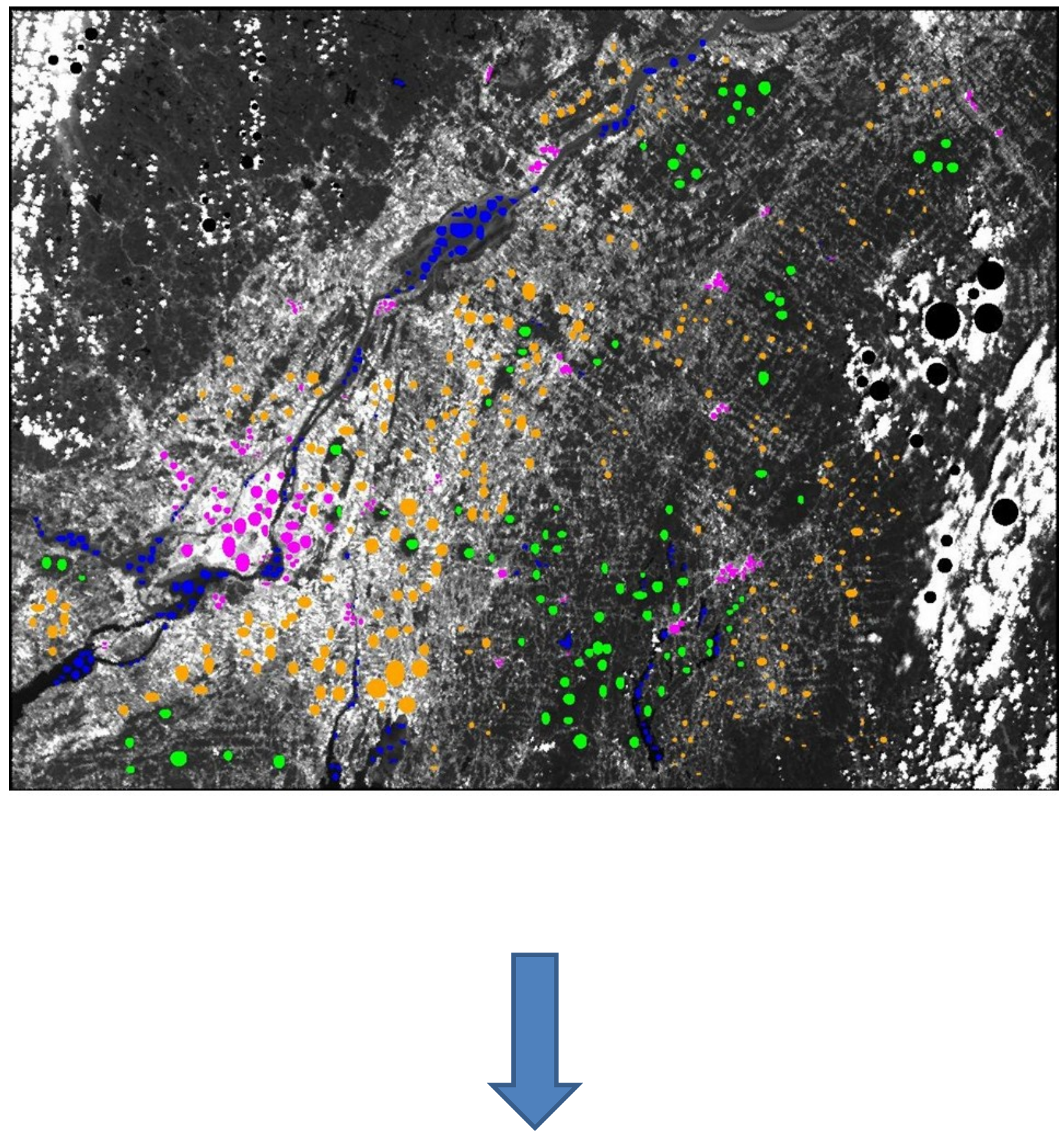


\section{c. Image classée}

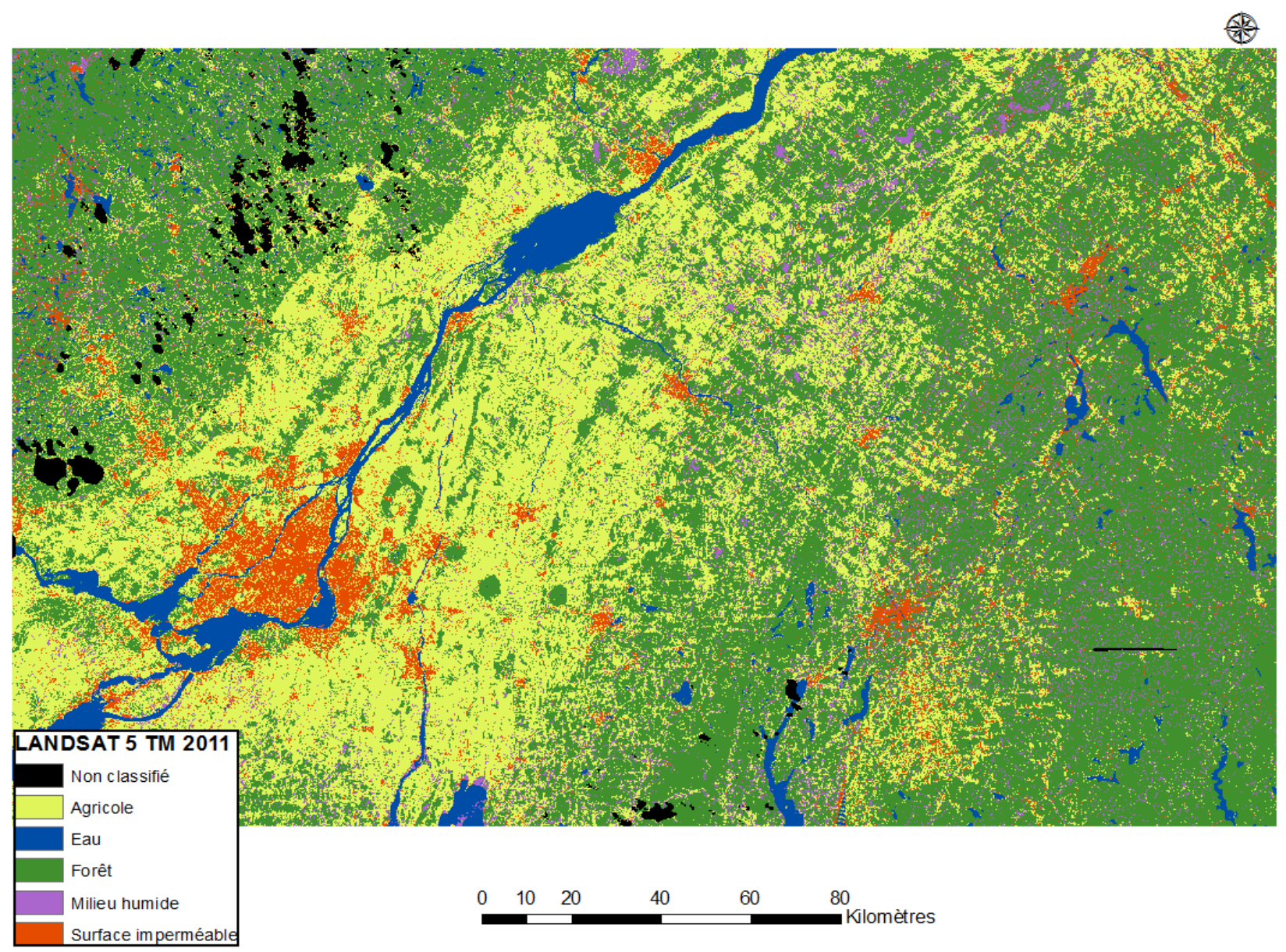

\section{Analyses statistiques}

Pour chaque capteur, nous avons effectué des régressions logistiques afin d'évaluer la relation entre la concentration moyenne en coliformes et les facteurs environnementaux.

\section{Résultats}

\section{Efficacité de l'imagerie satellite à caractériser le territoire}

La classification des images satellites a donné de bons résultats pour la zone d'étude, mais les estimations des surfaces correspondant aux déterminants environnementaux étudiés dépendaient fortement du type d'image satellite utilisé.

\section{Analyses statistiques}

À l'aide d'analyses de régression logistique, nous avons repéré deux utilisations des terres associées à un risque accru de forte concentration de coliformes fécaux : 1) les activités agricoles et 2) les activités urbaines. Parmi les capteurs étudiés, Landsat- 5 serait le plus performant pour ce qui est de la qualité de l'ajustement du modèle et de la capacité du modèle à classer les plages dans la bonne catégorie. 


\section{Comparaison des images d'observation de la Terre}

On a comparé plusieurs types d'images satellites utilisées dans le cadre de cette étude à l'aide des divers critères opérationnels mentionnés précédemment. Ces comparaisons ont révélé que le capteur Landsat- 5 constituait le meilleur compromis entre l'ensemble des critères, notamment parce qu'il offre les meilleures performances statistiques et fournit gratuitement les images.

\section{Discussion}

Ce projet a montré que les données extraites des images satellites pouvaient être utilisées pour caractériser les zones régionales et déterminer les facteurs environnementaux qui font augmenter le risque de contamination fécale des eaux récréatives. Le repérage des plages présentant un plus fort risque de contamination fécale est un élément important d'une évaluation globale de la qualité microbiologique de l'eau, en intégrant à la fois le risque et le niveau actuel de pollution fécale. En repérant au préalable les endroits à risque élevé et en déterminant la vulnérabilité environnementale potentielle des plages à la contamination fécale, cette approche pourrait contribuer à améliorer l'allocation des ressources pour les activités d'évaluation des risques, en particulier lorsqu'il s'agit de vastes territoires nécessitant la surveillance de centaines de plages.

Il est possible d'utiliser d'autres sources de données en plus de l'imagerie satellite pour caractériser l'environnement. On a utilisé les recensements, les enquêtes et les données de terrain à de nombreuses reprises; ces méthodes ont fait leurs preuves. Toutefois, en comparaison, l'imagerie satellite pourrait se révéler plus avantageuse. II convient également de prendre en compte certaines limites avant d'avoir recours à cette méthode à plus grande échelle dans les programmes de surveillance des eaux récréatives. Le Tableau 1 résume les avantages et les inconvénients de l'utilisation de l'imagerie satellite pour détecter les eaux récréatives où le risque de contamination fécale est le plus élevé.

\section{Tableau 1 : Avantages et inconvénients liés à l'utilisation de l'imagerie satellite pour détecter les} eaux récréatives exposées à un risque de contamination fécale.

\begin{tabular}{l|l} 
Avantages & Inconvénients \\
\hline $\begin{array}{l}\text { Offre une couverture considérable, y } \\
\text { compris celle des régions difficiles à } \\
\text { atteindre }\end{array}$ & $\begin{array}{l}\text { Nécessite une formation technique pour recueillir, traiter et } \\
\text { analyser les données }\end{array}$ \\
\hline Reproductibilité & Peu de sensibilisation dans le secteur de la santé publique \\
\hline Constance & Coût de certaines images \\
\hline Précision & $\begin{array}{l}\text { Incapacité de certains capteurs à enregistrer des données par } \\
\text { temps couvert }\end{array}$
\end{tabular}

Ce projet illustre l'une des nombreuses façons dont les technologies satellitaires peuvent aider à améliorer la santé publique. En effet, la qualité technique et la fiabilité opérationnelle actuelles des satellites de télédétection disponibles rendent leur utilisation pertinente et efficace pour faire face à différents enjeux de santé publique, dont les populations vulnérables dans les régions éloignées, les urgences de santé publique, les problèmes de sécurité sanitaire (p. ex. une catastrophe naturelle ou une éclosion de maladie) et les maladies qui dépendent de l'environnement comme celles transmises par les insectes ou la faune. Globalement, on considère aujourd'hui que les technologies spatiales sont des solutions abouties et flexibles qui peuvent considérablement renforcer notre capacité à atteindre les principaux objectifs de santé publique, dont la surveillance de la santé, l'évaluation des risques, et les mesures et interventions d'urgence. 


\section{Remerciements}

Les auteurs remercient les personnes participant au programme provincial de surveillance des eaux récréatives du Québec (Environnement-Plage) et toutes les personnes ayant contribué au rapport du projet (7). Les auteurs remercient également Guy Aubé et Paul Brian, de l'Agence spatiale canadienne, pour leur soutien tout au long de ce projet.

\section{Conflit d'intérêts}

II n'y a aucun conflit d'intérêts à déclarer.

\section{Financement}

Ce projet a été financé par l'Agence spatiale canadienne et l'Agence de la santé publique du Canada.

\section{Références}

(1) World Health Organization. Guidelines for safe recreationnal water environments. Coastal and fresh waters. Geneva, Switzerland: World Health Organization; 2003.

(2) Fleisher JM, Fleming LE, Solo-Gabriele HM, Kish JK, Sinigalliano CD, Plano L, Elmir SM, Wang JD, Withum K, Shibata T, Gidley ML, Abdelzaher A, He GQ, Ortega C, Zhu XF, Wright M, Hollenbeck J, Backer LC. The BEACHES Study: health effects and exposures from non-point source microbial contaminants in subtropical recreational marine waters. Int J Epidemiol. 2010; 39(5):1291-8.

(3) Pruss A. Review of epidemiological studies on health effects from exposure to recreational water. Int J Epidemiol. 1998; 27(1):1-9.

(4) Pond K. Water recreation and disease, plausibility of associated infections: acute effects, sequelae and mortality. London: IWA Publishing; 2005.

(5) Marechal F, Ribeiro N, Lafaye M, Güell A. Satellite imaging and vector-borne diseases: the approach of the French National Space Agency (CNES). Geospatial health. 2008; 3(1):1-5.

(6) Turgeon P, Michel P, Levallois P, Ravel A, Archambault M, Lavigne M-P, Kotchi SO, Barzeau S. Assessing and monitoring agroenvironmental determinants of recreational freshwater quality using remote sensing. Water Science and Technology. 2013; 67(7):1503-11.

(7) Public Health Agency of Canada. Risk assessment of microbial contamination of recreational waters in Canada using satellite imagery: pilot project on public beaches in southern Quebec. Report No.: ISBN:978-1-100-54478-6. Saint-Hyacinthe: Public Health Risk Sciences Division of the Laboratory for Foodborne Zoonoses; 2013.

(8) Programme Environnement-Plage (base de données en ligne). Ministère du Développement durable, de l'Environnement et des Parcs du Québec; 2006.

Accès : http://www.mddep.gouv.qc.ca/programmes/env-plage/

(9) MDDEP. Guide d'application du programme Environnement-Plage. Québec : ministère du Développement durable, de l'Environnement et des Parcs du Québec; 2005. 
(10) Crowther J, Kay D, Wyer MD. Faecal-indicator concentrations in waters draining lowland pastoral catchments in the UK: relationships with land use and farming practices. Water Res. 2002; 36(7):1725-34.

(11) Haack SK, Fogarty LR, Wright C. Escherichia coli and enterococci at beaches in the Grand Traverse Bay, Lake Michigan: sources, characteristics and environmental pathways. Environ Sci Technol. 2003; 37(15):3275-82.

(12) Love DC, Lovelace GL, Money ES, Sobsey MD. Microbial fecal indicator concentrations in water and their correlation to envionmental parameters in nine geographically diverse estuaries. Water Qual Expo Health. 2010; 2:85-95.

(13) Marsalek J, Rochfort Q. Urban wet-weather flows: sources of fecal contamination impacting on recreational waters and threatening drinking-water sources. J Toxicol Environ Health A. 2004; 67(20-22):1765-77.

(14) Olyphant GA, Whitman RL. Elements of a predictive model for determining beach closures on a real time basis: The case of 63rd Street Beach Chicago. Environ Monit Assess. 2004; 98(13):175-90.

(15) Turgeon P, Michel P, Levallois P, Archambault M, Ravel A. Fecal contamination of recreational freshwaters: the effect of time-independent agroenvironmental factors. Water Qual Expo Health. 2011; 3(2):109-18.

(16) Ramos MC, Quinton JN, Tyrrel SF. Effects of cattle manure on erosion rates and runoff water pollution by faecal coliforms. J Environ Manage. 2006; 78(1):97-101.

(17) Rodgers P, Soulsby C, Hunter C, Petry J. Spatial and temporal bacterial quality of a lowland agricultural stream in northeast Scotland. Sci Total Environ. 2003; 314-316:289-302.

(18) Kao C, Wu M. Control of non-point source pollution by a natural wetlands. Water Sci Technol. 2001; 43(5):169-74.

(19) Knox AK, Dahlgren AR, Tate KW, Atwill ER. Efficacy of natural wetlands to retain nutrient, sediment and microbial polluants. J Environ Qual. 2008; 37:1837-46.

(20) Matteo M, Randhir T, Bloniarz D. Watershed-scale impacts of forest buffers on water quality and runoff in urbanizing environment. J Water Res PI Manag. 2006; 132(3):144-52.

(21) McKenney DW, Hutchinson MF, Kesteven JL, Venier LA. Canada's plant hardiness zones revisited using modern climate interpolation techniques. Can J Plant Sci. 2001; 81:129-43.

(22) Site sur la rusticité des plantes du Canada (base de données en ligne). Ressources naturelles Canada. 2007. Accès : http://planthardiness.gc.ca/index.pl?m=14\&.\&\&lang=fr 